The study aims to compare long-term effects of artificial lung ventilation sustained in the neonatal period in dependence on newborn babies` gestational age and the mechanical parameters of ventilation.

Materials 127 patients aged 8-11 (84 full-term babies, 43 premature babies) who were treated in the intensive care unit of St. Petersburg Children`s Hospital No. 1 in the neonatal period, underwent follow-up examination of respiratory system. Of them, 27 children were born with the gestational age of $30-34$ weeks, 16 of $35-36$ weeks, and 84 at the gestational age of $37-42$ weeks. The control group consisted of 43 children with uncomplicated neonatal period.

Results In the neonatal period, pathology of the respiratory system was detected much more often in the premature infants $(56 \%$ and $88 \% ; p<0.001)$. They developed 1 st type respiratory distress syndrome $(45 \%)$ more often than the fullterm infants, while the amniotic fluid aspiration syndrome was detected in the full-term newborns more often than in the premature ones (36\%). Transient tachypnea of the newborn was significantly more frequent in the group of full-term infants $(28 \%$ and $8 \% ; \mathrm{p}=0,026)$. Among infants with bronchopulmonary pathology, pneumonia developed in preterm babies more often $(32 \%$ and $60 \% ; \mathrm{p}=0.009)$. According to a follow-up survey, the incidence of pulmonary healthy children was comparable in the group of full-term children $(74 \%)$ and in the control group $(84 \%)$ at school age, while prematurely born children developed no bronchopulmonary pathology less often $(63 \%, p=0.05)$. Besides, prematurely born children with recurrent bronchitis developed neonatal pneumonia reliably more often $(\mathrm{p}<0.05)$. All prematurely born children who developed recurrent bronchitis or chronic nonspecific lung diseases at school age (100\%) had been on ALV with 'hard' settings in the neonatal period, whereas the same indicator among full-term infants was twice lower (50\%). Family history analysis showed that family history of bronchopulmonary diseases accompanied bronchial asthma in most cases, both in full-term (67\%) and premature children (63\%).

Conclusions Neonatal pneumonia, along with iatrogenic effects of resuscitation, is the dominant factor in formation of chronic nonspecific pulmonary diseases in catamnesis. Children treated in ICU in the early neonatal period should be considered a high-risk group for development of bronchopulmonary system pathology later in life, and they must be carefully supervised by pediatrician and pulmonologist.

\section{P552 BRITISH THORACIC SOCIETY (BTS) PNEUMONIA AUDIT: COMPLETING THE LOOP}

Ehtasham Yousaf*, M Shahid Yousuf, Catalin Soroiu, Mohammad Tariq. Midlands Regional Hospital, Portlaoise, Ireland

\subsection{6/archdischild-2019-epa.886}

Aims Our aim was to collect data on children admitted to Midlands Regional Hospital, Portlaoise (MRHP) with a diagnosis of Community Acquired Pneumonia (CAP). We looked into presenting complaints, clinical signs, investigation, management and follow-up plan made on these patients. In winter 2016/17 this data was compared with data from 16 other hospitals across the UK and Ireland as part of the British Thoracic Society's (BTS) audit on CAP. Now, in winter 2018/
19, we did re-audit to look at progress of our own department in 2 years.

Methods A retrospective chart review was carried out to identify patients with CAP. Inclusion criteria included children presenting to MRHP aged 1-15 years with a diagnosis of CAP over Oct Dec 2018.

Results There was a massive improvement regarding choice of antibiotics to treat CAP and we switched to amoxicillin in majority of cases, which is in line with recommendation of BTS, UK. Our data is comparable to international data and previous local data with regard to demographics and severity of pneumonia requiring $\mathrm{O} 2$ therapy. We still are performing more blood tests (including acute phase reactants and microbiological investigations) and chest $\mathrm{x}$-rays than other centers but there is decrease in these numbers as compared to our own previous data.

Conclusions The over diagnosis, over investigation and over treatment of CAP is leading to wasted resources and is not in adherence with best practice. After the recommendations from our first audit, there was big improvement in few domains and we are progressing towards best practice in others. We are also in the process of completion of our local guidelines and after their introduction; we expect our efficacy to improve even more.

\section{REFERENCES}

1. BTS UK community acquired pneumonia in children guidelines: https://www.britthoracic.org.uk/document-library/audit-and-quality-improvement/audit-reports/btspaediatric-community-acquired-pneumonia-audit-report-201617/https://www.britthoracic.org.uk/document-library/clinical-information/pneumonia/paediatric-pneumonia/bts-guidelines-for-the-management-of-community-acquired-pneumonia-in-children-update-2011/

\section{P553 IMPACT OF THE INTERNATIONAL GUIDELINES ON THE THERAPY OF BRONCHIOLITIS: SINGLE-CENTRE RETROSPECTIVE ANALYSIS}

Veronika Csuzdi*, Gábor Uhereczky, Éva Gács, G Ágnes Tóth, Gabriella Stéger, László Szabó. Heim Pál National Paediatric Institute, Budapest, Hungary

\subsection{6/archdischild-2019-epa.887}

The AAP bronchiolitis guidelines published in 2006 and updated in 2014 recommended supportive care with limited diagnostic testing and treatment. To comply with the international guidelines in 2014 we've started a campaign to improve the therapeutic practice of bronchiolitis at our hospital.

Aim To assess the effect of the therapeutic regime changes on the morbidity of bronchiolitis (need of PICU transmission, length of hospital stay) at our hospital.

Methods The data of 225 patients, aged 1-12 months, hospitalized in the period of April to October between 2013 and 2018 with the diagnosis of bronchiolitis at our Pulmonology Unit was analysed retrospectively.

Results Antibiotic treatment application decreased since 2013 in each year (In 80\%-36\%-27\%-12\%-2\% of the patients respectively). Same as the antibiotic use, the systemic corticosteroid and inhalative B2 agonist administration decreased significantly $(60 \%-28 \%-17 \%-2 \%-0 \%$ and $80 \%-39 \%$ $-40 \%-13 \%-14 \%$ of the cases). Use of $3 \%$ saline inhalation increased: $30 \%-39 \%-93 \%-93 \%-95 \%$. Although the use of drug therapies decreased during the observational period, it did not cause an increase either in the length of hospital stay (median days $6.4-6.3-6.9-4.5-5.0$ ) or in 